\title{
Protocolo de activación quirúrgica COVID-19 (PAQ COVID)
}

\section{Surgical activation protocol COVID-19 (SAP COVID)}

\author{
Abelardo Juanz-González', Jorge A. Barreras-Espinoza', Eduardo Leyva-Moraga *, \\ Francisco A. Leyva-Moraga² y Fernando Leyva-Moraga² \\ ${ }^{1}$ Departamento de Cirugía, Hospital General del Estado de Sonora; '2Departamento de Medicina y Ciencias de la Salud, Universidad de \\ Hermosillo, Sonora, México
}

\section{Resumen}

Antecedentes: La pandemia de COVID-19 ha tenido un gran impacto en la práctica quirúrgica convencional. Conforme el número de pacientes diagnosticados con esta enfermedad vaya en aumento, el riesgo de contagio para el equipo quirúrgico será mayor. Pocas publicaciones han abordado el manejo del paciente diagnosticado con COVID-19 dentro del quirófano. Objetivo: Evaluar las recomendaciones para el cuidado de pacientes y del equipo quirúrgico durante la pandemia de CoVID-19. Método: La búsqueda bibliográfica principal utilizó las bases de datos MEDLINE, Embase y Cochrane, utilizandô las palabras clave "COVID-19", "PROTOCOL" y "SURGERY". Se verificaron recomendaciones, guías y series de casos relevantes para obtener la información más precisa y aplicable. Resultados: Se hizo la revisión de 379 artículos que contenían las palabras clave. Se incluyeron 25 artículos basándose en la pertinencia de las recomendaciones. Los tres temas principales seleccionados fueron las fases preoperatoria, transoperatoria y posoperatoria. Conclusión: En un esfuerzo por tratar de normar el manejo quirúrgico, presentamos recomendaciones para preservar la seguridad del paciente y del equipo quirúrgico con estándares de alta calidad, mediante estrategias reproducibles en la mayoría de los centros hospitalarios.

Palabras clave: Cirugía. COVID-19. Protocolo. Sala de operaciones.

\begin{abstract}
Background: The coronavirus disease 2019 (COVID-19) outbreak have major implications in conventional surgical practice. As the number of patients with this diagnosis is rising, the infection risk for the surgical staff will be higher. Few publications have addressed the surgical management of patients diagnosed with COVID-19. Objective: To assess recommendations for care of patients and surgical team during the COVID-19 pandemic. Method: MEDLINE, Embase and the Cochrane Datäbase of Systematic Reviews (April 2020) were searched the key words "COVID-19", "PROTOCOL" and "SURGERY". Relëvant recommendations, guidelines and cases series were checked for the most accurate information for apply to our center. Results: We found 379 papers that included the key words. A total of 25 papers were included in the manuscript based iR the pertinence of the recommendations. Three major topics were selected: perioperative, intraoperative and postoperative. Conclusion: As an attempt to regulate the surgical team approach, we present recommendations to preserve patientsaand surgical staff safety with high quality standards of care through reproducible strategies applicable in most hospital centers.
\end{abstract}

Key words: Surgery. COVID-19. Protocol. Operating room.

\section{Correspondencia:}

*Eduardo Leyva-Moraga

Avda. Luis Donaldo Colosio

Col. Centro

Fecha de recepción: 14-04-2020

C.P. 83000 , Hermosillo, Son., México

E-mail: leyvamoragaeduardo@gmail.com

0009-7411/0 2020 Academia Mexicana (http://creativecommons.org/licenses/by-nc-nd/4.0/).
Fecha de aceptación: 12-06-2020

DOI: 10.24875/CIRU.20000331
Cir Cir. 2021;89(1) $4-11$

Contents available at PubुMed www.cirugiaycirujanos.com www.cirugiaycirujanos.com
ajo la licencia CC BY-NC-ND 


\section{Introducción}

Hasta el día 12 de junio de 2020, 7,778,078 personas han sido contagiadas de COVID-19 en 188 países. En México se han confirmado 133,974 casos, dejando la estela de 15,944 muertes hasta la fecha'. La COVID-19 ha cambiado drásticamente la manera en que las instituciones manejan los servicios de cirugía. Los procedimientos electivos han sido pospuestos para priorizar las cirugías de emergencia y consecuentemente reducir el riesgo de exposición del equipo quirúrgico². Considerando el alcance de esta pandemia, es prudente pensar que las emergencias quirúrgicas en pacientes con sospecha o diagnóstico de COVID-19 serán cada vez más frecuentes. Es necesario reformular nuestra práctica convencional para continuar brindando una atención de calidad de manera segura para el equipo quirúrgico, haciendo uso adecuado y racional del equipo de protección personal (EPP) y de los insumos. En este artículo presentamos las estrategias fundamentales para la realización de intervenciones quirúrgicas en pacientes con diagnóstico o sospecha de COVID-19. Por diferencias económicas o de infraestructura, algunas recomendaciones podrán no ser directamente aplicables en todas las instituciones, pero su fundamento podrá ser adaptado según las circunstancias particulares de cada centro.

\section{Método}

\section{Revisión de la literatura}

Los autores se reunieron en marzo de 2020. Los temas para la inclusión de estudios fueron acordados y asignados. La búsqueda bibliográfica principal utilizó las bases de datos MEDLINE, Embase y Cochrane, para identificar las contribuciones relevantes publicadas desde enero de 2020 hasta la fecha. Se utilizaron los términos de encabezamientos de temas médicos (MESH), al igual que los términos de entrada. Las palabras clave fueron "COVID-19", "PROTOCOL", "OPERATING ROOM" y "SURGERY". Se verificaron las listas de referencias de todos los artículos elegibles.

\section{Selección de los estudios}

Los títulos y los resúmenes fueron seleccionados por revisores individuales para identificar artículos potencialmente relevantes. Las discrepancias en el juicio fueron resueltas por el autor principal durante las reuniones del comité del grupo de trabajo del protocolo de activación quirúrgica COVID-19 (PAQ COVID)

\section{Resultados}

\section{Transmisión del virus}

Las principales vías de transmisión del SARS-CoV 2 se resumen en dos: 1) inhalación de gotas de aerósol expulsadas al toser, hablar o respirar ${ }^{3,4}$, y 2) contaicto directo con superficies contaminadas, como el plástico y el acero inoxidable, donde el virus puede permanecer activo hasta 72 horas $^{5}$. Si bien se ha identificado la presencia de RNA viral en tejido extrapulmonar (materia fecal, sangre, tejido gastrointestinal y líquido peritoneal), no se ha comprobado formalmente una rutâ de transmisión adicional a la respiratoria ${ }^{6-8}$.

\section{Equipo de protección personal}

El EPP debe cubrir completamente la piel, los ojos, los orificios nasales y la boca. Los EPP incluyen guantes, bata impermeable, mascarillas FFP2/FFP3, lentes protectores, careta facial, guantes, gorio y botas. Todo el personal dentro de la sala de opêraciones deberá utilizar estas medidas de protecéión (Fig. 1) ${ }^{8}$. La colocación y el retiro del EPP deben supervisados, además de contar con una secuencia específica y un área destinada a ello ${ }^{8}$. Determinar los niveles de riesgo de contaminación cruzadå de COVID-19 disminuirá el uso inapropiado de los EPP.

\section{Secuencia de colocación y retiro del EPP} (donning-doffing)

Nosotros basamos nuestras recomendaciones para realizar la secuencia de colocación y retiro del EPP de acuerdo con la técnica descrita por Balibrea, et al. ${ }^{9}$. La figura 1 resume estas secuencias en guirófano. Entre el retiro de cada EPP se deberá realizar lavado clínico de manos con solución alcoholadau

\section{Características del quirófano COVID-19}

El riesgo de transmisión disminuye al utilizar elmismo quirófano y la misma máquina de anestesia ${ }^{10}$, jpor lo que se asigna un área de quirófanos exclusiva para pacientes con COVID-19. Sin embargo, en los centros donde esta conversión no sea posible, una sola šala se utilizará durante toda la pandemia. La salầ de operaciones debe ser de fácil acceso, con una ruta 
1. Doble gorro quirúrgico.

2. Mascarilla N95 o FFP2/FFP3, mascarilla quirúrgica estándar, gafas protectoras y careta facial.

3. Bata quirúrgica.

4. Guantes: (par de guantes de nitrilo y dos pares de guantes para el procedimiento).

5. Bata impermeable.

6. Botas quirúrgicas.

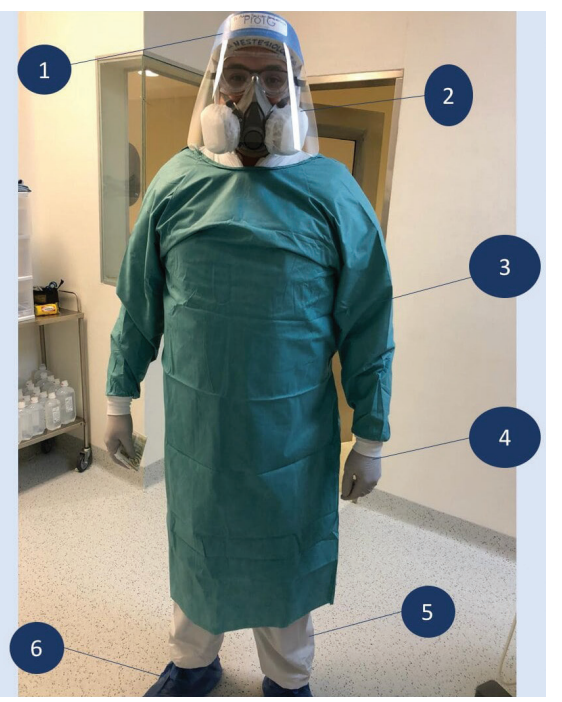

Figura 1. Equipo de protección personal.

Tabla 1. Tiempos de entrada y salida del equipo COVID

\begin{tabular}{|c|c|c|c|c|}
\hline Integrante & Entrada & Salida & EPP* & 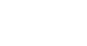 \\
\hline Camillero & $\begin{array}{l}\text { Encargado de llevar al paciente al sitio de } \\
\text { transferencia }\end{array}$ & $\begin{array}{l}\text { Encargado de llevar al paciente a zona de } \\
\text { aislamiento }\end{array}$ & Riesgo Il/full & $\frac{\mathrm{EPP}}{\frac{\mathrm{P}}{\mathrm{O}}}$ \\
\hline Enfermera circulante & $\begin{array}{l}\text { Recibe al paciente en la zona de } \\
\text { transferencia y es la encargada de llevar al } \\
\text { paciente al quirófano }\end{array}$ & $\begin{array}{l}\text { Activar el código de salida del paciente } \\
\text { Una vez recuperado el paciente, se encargará de } \\
\text { entregarlo en la zona de transferencia }\end{array}$ & $\begin{array}{l}\text { Riesgo III/enh } \\
\text { EPP }\end{array}$ & 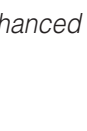 \\
\hline $\begin{array}{l}\text { Enfermera } \\
\text { instrumentista }\end{array}$ & $\begin{array}{l}\text { Ya que el paciente cuente con el } \\
\text { procedimiento por parte de anestesiología }\end{array}$ & Al terminar el procedimiento & $\begin{array}{l}\text { Riesgo III/enh } \\
\text { EPP }\end{array}$ & hanced \\
\hline Anestesiología & $\begin{array}{l}\text { Una vez que el paciente esté en la mesa } \\
\text { quirúrgica }\end{array}$ & Una vez que el paciente se recupere & $\begin{array}{l}\text { Riesgo III/enh } \\
\text { EPP }\end{array}$ & hanced \\
\hline Cirujanos & $\begin{array}{l}\text { Ya que el paciente cuente con el } \\
\text { procedimiento por parte de anestesiología }\end{array}$ & Al terminar el procedimiento & $\begin{array}{l}\text { Riesgo III/enh } \\
\text { EPP }\end{array}$ & hanced \\
\hline Personal de limpieza & Al momento de salir el paciente & Al terminar la desinfección de la sala de quirófano & Riesgo II/full & EPP \\
\hline
\end{tabular}

EPP: equipo de protección personal.

*Recomendaciones de la Organización Mundial de la Salud y de los Centers for Disease Control and Prevention†.

establecida y señalizada. Se recomienda el uso de sistema de presión negativa, y en caso de no contar con este, el sistema de flujo laminar con recambios de más de 20 ciclos/hora será una alternativa ${ }^{11}$.

\section{Fase preoperatoria}

1) Valoración preoperatoria del paciente

Se recomienda cancelar consultas no esenciales y procedimientos electivos, ya que se podría acelerar y exacerbar la progresión de la COVID-1912. En casô que el diferimiento de la cirugía prolongue la estañcia hospitalaria, aumente el riesgo de complicaciones $气$ el manejo médico haya fallado, deberá realizarse lacírugía de emergencia ${ }^{11}$.

Se consideran casos sospechosos los pacientes con cuadro clínico de tos, fiebre $\left(\geq 38^{\circ} \mathrm{C}\right.$ o $\left.100.4{ }^{\circ}{ }^{\circ} \mathrm{E}\right)$ y cefalea, que necesitan ser hospitalizados y que presenten signos clínicos o radiográficos de consolidāión no explicada por otra infección, y que en los últimos 10 

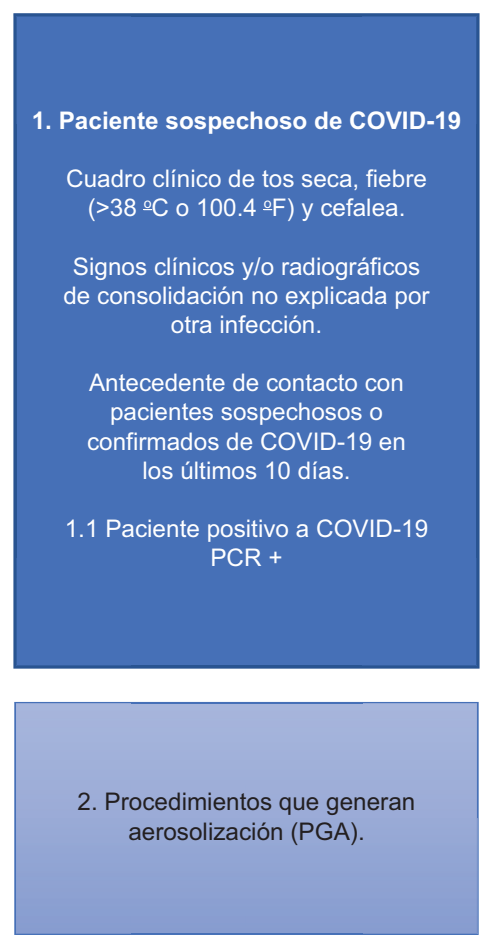

días presenten el antecedente epidemiológico de contacto con un caso probable o confirmado ${ }^{13}$ (Fig. 2).

La realización sistemática de una tomografía computada de tórax deberá considerarse como un apoyo diagnóstico para el paciente sintomático de COVID-19, pero hasta el momento no ha demostrado ser un estudio de primera línea ${ }^{14,15}$.

Se realiza también una anamnesis completa, con el propósito de identificar factores de riesgo para desarrollar enfermedad grave, tales como enfermedad pulmonar obstructiva crónica, hipertensión arterial, diabetes mellitus, enfermedad renal crónica ${ }^{16}$ o padecimientos oncológicos en pacientes que hayan sido operados o hayan recibido quimioterapia ${ }^{17}$. El uso de antiinflamatorios no esteroideos en etapas tempranas de la enfermedad se ha asociado a complicaciones cardiacas y pulmonares graves ${ }^{18}$.

2) Fase de preparación

Inicia una hora antes del procedimiento y durante este tiempo se desaloja la ruta y se restringe el acceso al personal. Los profesionales de enfermería del área de aislamiento son los responsables del traslado. En caso de encontrarse en la unidad de cuidados intensivos (UCI), el paciente deberá ser acompañado por el intensivista, quien lo llevarã directamente a la sala de operaciones. Se recomienda el uso de ventilador de traslado a los pacien̆tes que requieran ventilación mecánica; de no ser àsí, el paciente únicamente utilizará mascarilla N95 y gorro (el uso de la mascarilla con reservorio debe de ser evitado). Se recomienda, al momento del cambio de ventiladores, la oclusión del tubo endotraqueal ${ }^{19}$. El uso de sistemas cerrados de transp̄orte (cápsulas de aislamiento) (Fig. 3) no ha demostrado tener beneficio, pero en caso de con̆tar con este recurso podrá utilizarse debido a que-representa una barrera física adicional entre el personal y paciente. La enfermera circulante se encarga de mantener el acceso libre para el paciente, y a $\vec{a}$ su vez ayuda a la enfermera instrumentista a preparar y abrir la mesa quirúrgica antes del arribo. El servicio de anestesiología prepara el material necesario 


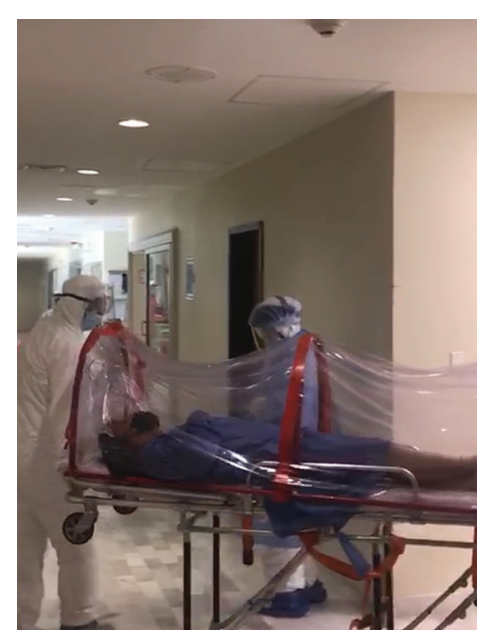

Figura 3. Traslado en cápsula de aislamiento.

y hace una segunda revisión de la máquina de anestesia en caso de requerirse inducción. Se sugiere hacer una doble revisión de los procedimientos de seguridad del paciente. Recomendamos el uso de la caja de aerosol para la intubación y la extubación.

\section{Fase intraoperatoria}

Este periodo comienza con la llegada del paciente a la sala de cirugía. Con el fin de disminuir el riesgo de exposición, recomendamos las siguientes estrategias:

\section{1) Equipo PAQ-COVID}

El equipo de trabajo deberá estar integrado por los siguientes participantes: enfermera circulante externa, enfermera circulante, instrumentista, anestesiólogo principal, ayudante de anestesiólogo, cirujano principal y cirujano ayudante. El total de las personas integrantes se asignará dependiendo del procedimiento quirúrgico. La entrada y la salida, así como el grado de riesgo, se resumen en la tabla 1.

2) Tipos de abordajes quirúrgicos

Se deben tomar en cuenta los riesgos de exposición en la toma de decisión entre cirugía abierta o laparoscópica; la evidencia está limitada a una serie de casos aprendidos de enfermedades víricas altamente transmisibles por fluidos ${ }^{10}$.

Por los beneficios que presentan las técnicas de mínima invasión, como disminuir el tiempo de estancia intrahospitalaria, ser una barrera física entre el cirujano y la posible fuente de contagio, no se descarta su uso. En caso de utilizarlas se recomienda lo siguiente:
- Utilizar presiones bajas de neumoperitoneo sin limitar la visión del campo quirúrgico ${ }^{11}$.

- Incisiones lo suficientemente pequeñas paradejar pasar el trocar y evitar la fuga de dióxidède carbono ${ }^{11}$.

- El neumoperitoneo debe de ser eliminado añtes de comenzar el cierre, retirar los trocares, sacar la pieza quirúrgica o convertir a procedimiento abierto, por medio de un circuito cerrado de eliminación.

3) Electrocirugía

El uso electrocirugía debe ser disminuido. De ser necesario, se recomienda utilizar aspirador de hưmo y titular los niveles más bajos de energía ${ }^{20,21}$.

4) Anestesia general versus regional o local

Es recomendable la anestesia regional o localsobre la general ${ }^{22}$. En caso de intubación endotraqưeal se prefieren las secuencias rápidas de intubación realizadas por un experto ${ }^{23}$. Se recomienda el cuso de caja de aerosol para intubación de secueñcia rápida y extubación. Se sugiere aplicar alcohol â la línea intravenosa o los catéteres intravenosos y ålos tripies $^{24}$.

5) Caja de aerosol

La caja de aerosol es un dispositivo no hermétitico, hecho a base de acrílico o policarbonato, que actúa como una barrera adicional entre el médico que liza la secuencia de intubación rápida y el paciente ${ }^{25,26}$. Se coloca sobre la cama quirúrgica, cubriendo la región cefálica con el objetivo de disminuir el contacto directo con aerosoles emitidos al respirar toser o hablar. Además, es posible reutilizar la caja de-aerosoles para otros procedimientos después de sưcorrecta desinfección con alcohol al $70 \%$ o ctoro $(5000 \mathrm{mg} / \mathrm{l})(\text { Figs. } 4 \text { y } 5)^{27}$.

En caso de que la caja represente un obstáculo para la secuencia rápida o la extubación, sugerimos retirarla. Recomendamos utilizar un videolaringoscopio para disminuir el rango de movimientos requerielos durante la manipulación de la vía aérea. Utilizar esta técnica puede implicar un riesgo en caso de no disponer de personal entrenado al respecto. Hastä el momento no se cuenta con evidencia de su eficacia, pero consideramos que es una medida adecuăda para la protección del equipo PAQ COVID.

6) Procedimientos con alto riesgo de aerosolización

Las traqueostomías, las endoscopias y las broncoscopias deben ser manejadas como pacientes $\check{C O}$ VID-19 confirmados, extremando el uso de los EPP22.

Según las guías de Ear, Nose and Throat surge in the United Kindom (ENT UK), la traqueostomía es 


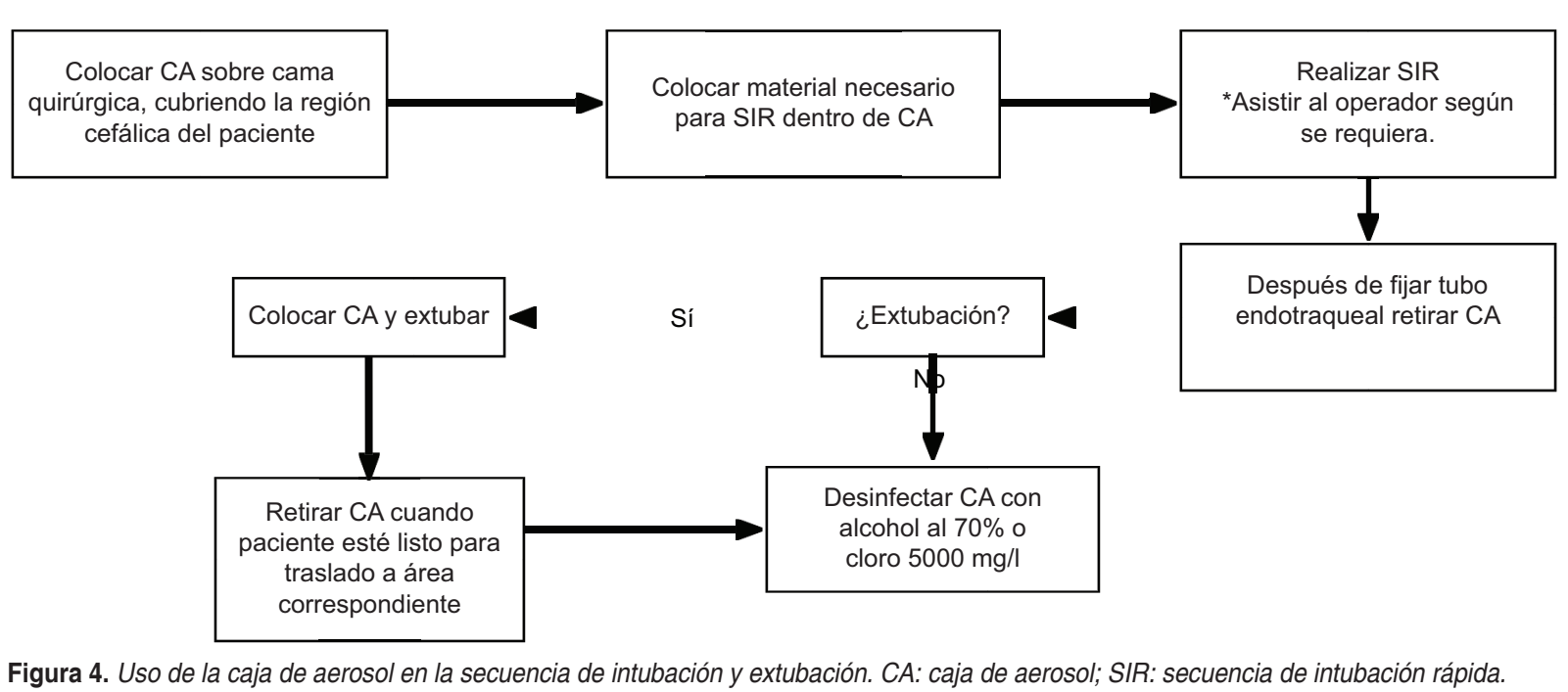

Figura 4. Uso de la caja de aerosol en la secuencia de intubación y extubación. CA: caja de aerosol; SIR: secuencia de intubación rápida

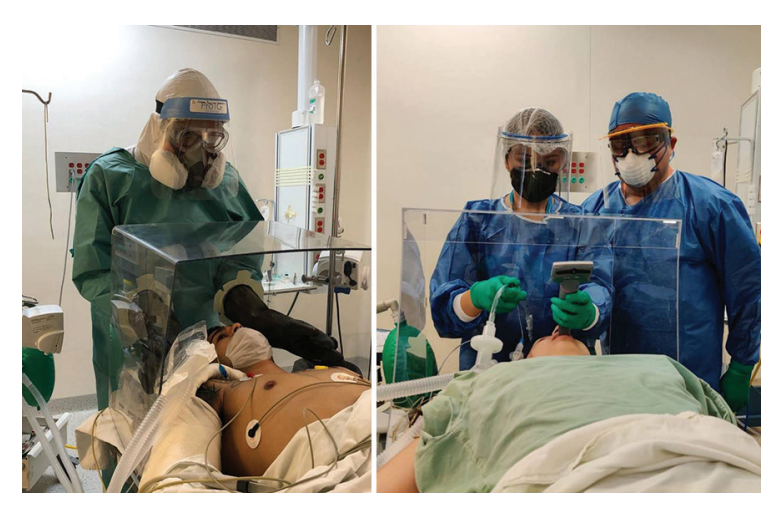

Figura 5. Secuencia de intubación rápida con la caja de aerosol.

un procedimiento electivo y conlleva un riesgo específico, por lo que se requiere un manejo individualizado ${ }^{28}$. Hasta el momento se cuenta únicamente con dispositivos prototipo, que permiten mantener un circuito cerrado de la vía aérea y a su vez la introducción del endoscopio ${ }^{29}$.

\section{Fase posoperatoria}

Inicia al finalizar el procedimiento quirúrgico, pero media hora antes del término de la cirugía debe ser activado el traslado. La recuperación anestésica se llevará a cabo en el quirófano. En caso de requerir ventilación mecánica, el paciente egresará directamente a la UCI acompañado por la enfermera circulante y el equipo de anestesiología ${ }^{19}$.

1) Manejo de muestras, sangre y fluidos corporales
Las muestras o piezas quirúrgicas se colocan en doble bolsa y se envían directamente al laboratốrio. Para el manejo de derrames de sangre menores dé-10 $\mathrm{ml}$ se eliminan con toallas que contengan una solución clorada de $5,000 \mathrm{mg} / \mathrm{l}$ y posteriormente se realiza la limpieza de la superficie en dos ocasiones. Los derrames mayores de $10 \mathrm{ml}$ se cubren completamente compresas absorbentes, después se impregna conssolución clorada de $10,000 \mathrm{mg} / \mathrm{l}$ por 30 minutos cơmo mínimo y luego se retira con cuidado de no contaminnar otras superficies.

La materia fecal y el vómito se desinfectan añtes de ser desechados al drenaje colocándolos en un contenedor con una solución clorada de 20,000 m/l con una relación de 1:2 (desechos: solución desinfectante) por un periodo de 2 horas $^{30}$.

2) Manejo del instrumental quirúrgico y aseo del quirófano

El material y los medicamentos utilizados durante el procedimiento deben ser desechados. El instrumental y el material quirúrgico reutilizables se desinfectan con solución clorada (1,000 mg/l) por media hora. Despứés, de forma separada, se esteriliza el instrumentak de pacientes sin diagnóstico de COVID-19. Posteriơr al retiro de contaminantes visibles se realiza aseo de techo, paredes y piso con solución clorada de $1,000 \mathrm{mg} / \mathrm{ml}$ por un mínimo de 30 minutos, al igual que las superficies de monitores, camas y tripies. La sala de operaciones debe ser desinfectada por personal capacitado en el uso de EPP ${ }^{30}$. Se requiere esperar 1 hora cömo mínimo para hacer uso de la sala de operaciones después de terminar el procedimiento, tiempo que permite la limpieza y la preparación adecuadas de la sala. 


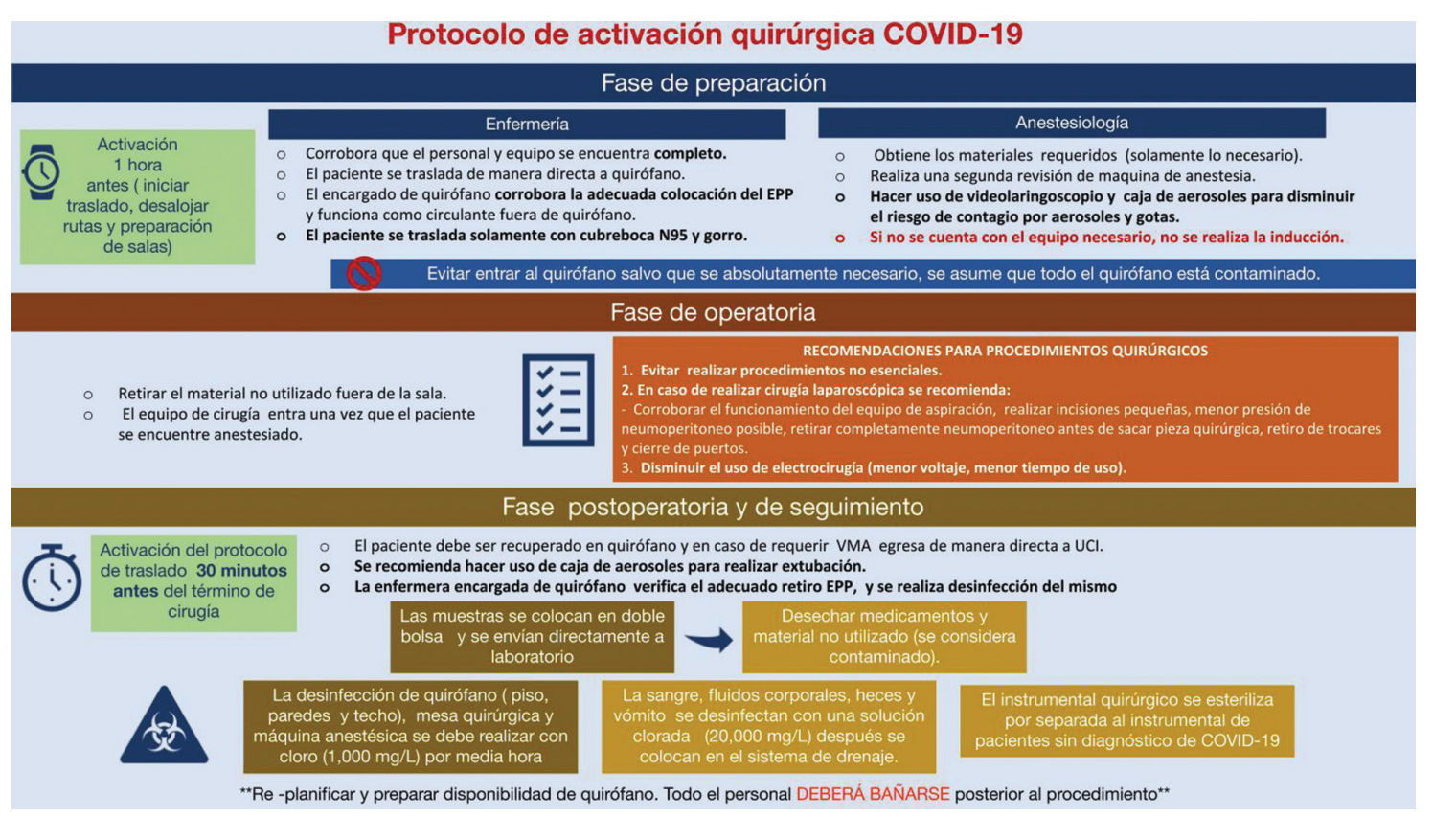

Figura 6. Resumen del protocolo de activación quirúrgica COVID-19.

\section{3) Seguimiento posoperatorio}

El seguimiento posoperatorio se realiza en el área de aislamiento o en la $\mathrm{UCl}$, dependiendo de la condición respiratoria del paciente; de preferencia, en un cuarto aislado y restringiendo el acceso a visitas. $\mathrm{Si}$ las condiciones generales del paciente lo permiten, se debe evitar todo contacto no esencial para su valoración posquirúrgica. Al egreso se realizarán consultas telefónicas o por videollamada ${ }^{16}$. El resumen de las fases se encuentra en la figura 6 .

\section{Conclusión}

A pesar de las adversidades de esta pandemia y de los grandes desafíos que tienen los centros hospitalarios, es imperativo que la práctica quirúrgica permanezca enfocada en la calidad de la atención y en la seguridad del paciente, así como en la del personal médico.

Las estrategias presentadas en este trabajo son reproducibles, accesibles y aplicables, considerando las limitaciones de la mayoría de las instituciones. Somos conscientes de que estas recomendaciones no son absolutas y deben adaptarse de manera dinámica a medida que se genere más conocimiento sobre el comportamiento de la COVID-19. Tenemos la certeza de que compartiendo nuestra perspectiva aportamos beneficios para el personal de salud y el manejo de los pacientes afectados.

\section{Conflicto de intereses}

Los autores certifican no estar involucrados en guna organización con intereses financieros o no financieros en la materia discutida en el manuscrito.

\section{Financiamiento}

No se recibió ningún tipo de financiamiento para la realización de este manuscrito.

\section{Responsabilidades éticas}

Protección de personas y animales. Los autơres declaran que para esta investigación no se han rèalizado experimentos en seres humanos ni en animales.

Confidencialidad de los datos. Los autores declaran que han seguido los protocolos de su centro de trabajo sobre la publicación de datos de pacientes.

Derecho a la privacidad y consentimiento informado. Los autores han obtenido el consentimiênto informado de los pacientes y/o sujetos referidos en el artículo. Este documento obra en poder del autor de correspondencia.

\section{Bibliografía}

1. COVID-19 Map. Johns Hopkins Coronavirus Resource Center. $\overline{2} 020$. (Consultado el 12 de junio de 2020.) Disponible en: https://coronavirus. jhu.edu/map.html 
2. McBride K, Brown K, Fisher O, Steffens D, Yeo D, Koh C. Impact of the COVID-19 pandemic on surgical services: early experiences at a nominated COVID-19 centre. ANZ Journal of Surgery. 2020;90:663-5.

3. Rothan H, Byrareddy S. The epidemiology and pathogenesis of coronavirus disease (COVID-19) outbreak. J Autoimmun. 2020;109:102433.

4. Bourouiba $L$. Turbulent gas clouds and respiratory pathogen emissions: potential implications for reducing transmission of COVID-19. JAMA 2020 Mar 26. doi: 10.1001/jama.2020.4756. Online ahead of print.

5. Van Doremalen N, Bushmaker T, Morris D, Holbrook M, Gamble A, Williamson B, et al. Aerosol and surface stability of SARS-CoV-2 as compared with SARS-CoV-1. N Engl J Med. 2020;382:1564-7.

6. Tian Y, Rong L, Nian W, He Y. Review article: gastrointestinal features in COVID-19 and the possibility of faecal transmission. Alim Pharmacol Ther. 2020;51:843-51.

7. Gu J, Han B, Wang J. COVID-19: gastrointestinal manifestations and potential fecal-oral transmission. Gastroenterology. 2020;158:1518-9.

8. Brat G, Hersey S, Chhabra K, Gupta A, Scott J. Protecting surgical teams during the COVID-19 outbreak: a narrative review and clinical considerations. Ann Surg. 2020 Apr 17:10.1097/SLA.0000000000003926. doi: 10.1097/SLA.0000000000003926. Online ahead of print.

9. Balibrea J, Badia J, Rubio Pérez I, Martín Antona E, Álvarez Peña E, García Botella S, et al. Manejo quirúrgico de pacientes con infección por COVID-19. Recomendaciones de la Asociación Española de Cirujanos. Cir Esp. 2020;98:251-9.

10. Pryor A. SAGES and EAES recommendations regarding surgical response to COVID-19 crisis. SAGES. 2020. (Consultado el 12 de junio de 2020.) Disponible en: https://www.sages.org/recommendations-surgical-response-covid-19/

11. Wong J, Goh Q, Tan Z, Lie S, Tay $Y, \mathrm{Ng} \mathrm{S}_{\text {S }}$ et al. Preparing for a COVID-19 pandemic: a review of operating room outbreak response measures in a large tertiary hospital in Singapore. Can J Anaesth. 2020;67:732-45.

12. Lei S, Jiang F, Su W, Chen C, Chen J, Mei W, et al. Clinical characteristics and outcomes of patients undergoing surgeries during the incubation period of COVID-19 infection. EClinicalMedicine. 2020;21:100331.

13. OMS. Definición para la identificación de los casos de enfermedad respiratoria grave asociada al nuevo coronavirus. Who.int. 2020. (Consultado el 12 de junio de 2020.) Disponible en: https://www.who.int/csr/ disease/coronavirus infections/case definition 2509 2012/es/

14. Ai T, Yang Z, Hou H, Zhan C, Chen C, Lv W, et al. Correlation of chest CT and RT-PCR testing in coronavirus disease 2019 (COVID-19) in China: a report of 1014 cases. Radiology. 2020 Feb 26:200642. doi: 10.1148/radiol.2020200642. Online ahead of print.

15. ACR Recommendations for the use of chest radiography and computed tomography (CT) for suspected COVID-19 infection. Acr.org. 2020. (Consultado el 12 de junio de 2020.) Disponible en: https://www.acr.org/Advocacy-and-Economics/ACR-Position-Statements/Recommendations-for-Chest-Radiography-and-CT-for-Suspected-COVID19-Infection.
16. Zhou F, Yu T, Du R, Fan G, Liu Y, Liu Z, et al. Clinical course and risk factors for mortality of adult inpatients with COVID-19 in Wuhan, China: a retrospective cohort study. Lancet. 2020;395:1054-62.

17. Tuech J, Gangloff A, Di Fiore F, Michel P, Brigand C, Slim K, et al. Strategy for the practice of digestive and oncological surgery during the Covid-19 epidemic. J Visc Surg. 2020 Jun;157(3S1):S7-S12.

18. Viswanath A, Monga P. Working through the COVID-19 outbreak ?rapid review and recommendations for MSK and allied health personnel. E Clin Orthop Trauma. 2020;11:500-3

19. Ti L, Ang L, Foong T, Ng B. What we do when a COVID-19 patientmeeds an operation: operating room preparation and guidance. Can J Anaesth. 2020;67:756-8.

20. American College of Surgeons. COVID 19: Considerations for Optimum Surgeon Protection. Before, During, and After Operation. Facs.org.2020. (Consultado el 12 de junio de 2020.) Disponible en: https://www.facs.org/-/ media/files/covid19/considerations_optimum_surgeon_protection.ashx.

21. NHS. Important and urgent - next steps on NHS response to COVID-19. NHS. 2020. (Consultado el 12 de junio de 2020.) Disponible en: https:// www.england.nhs.uk/coronavirus/wp-content/uploads/sites/52/2020/03/ urgent-next-steps-on-nhs-response-to-covid-19-letter-simon-stevens.pdf.

22. Forrester J, Nassar A, Maggio P, Hawn M. Precautions for operating room team members during the COVID-19 pandemic. J Am CollSurg. 2020;230:1098-101.

23. Kamming D, Gardam M, Chung FI. Anaesthesia and SARS. Br J Anaest. 2003:90:715-8.

24. Dexter F, Parra M, Brown J, Loftus R. Perioperative COVID-19 defense: an evidence-based approach for optimization of infection controf and operating room management. Anesth Analg. 2020;131:37-42.

25. Aerosol Box. Protects healthcare providers during endotracheal intubation. 2020. (Consultado el 12 de junio de 2020.) Disponible en: https:// sites.google.com/view/aerosolbox/design

26. Canelli R, Connor C, Gonzalez M, Nozari A, Ortega R. Barrier enčlosure during endotracheal intubation. N Engl J Med. 2020;382:1957-8.

27. Leyva Moraga F, Leyva Moraga E, Leyva Moraga F, Juanz GonzáTez A, Ibarra Celaya J, Ocejo Gallegos J, et al. Aerosol box, an operatingroom security measure in COVID-19 pandemic. World J Surg. 2020; 44:2049-50.

28. Harrison L. Tracheostomy guidance during the COVID-19 pandēmic. Entuk.org. 2020. (Consultado el 7 de abril de 2020.) Disponible éd: https://www.entuk.org/tracheostomy-guidance-during-covid-19-pandemic.

29. Marchese M, Capannolo A, Lombardi L, Di Carlo M, Marinangeli F, Fusco P. Use of a modified ventilation mask to avoid aerosolizing spread of droplets for short endoscopic procedures during coronavirus-COVID-19 outbreak. Gastrointest Endosc. 2020 Apr 2:S0016-5107(20)341316. doi: 10.1016/j.gie.2020.03.3853. Online ahead of print.

30. Liang T. Handbook of COVID-19 prevention and treatment. 2020. Item 1000/25 | Repositorio nacional [Internet]. 2020 [citado 25 Octubre 2020]. Disponible en: https://covid-19.conacyt.mx/jspui/handle/1000/25 